\title{
Modification of argon impurity transport by electron cyclotron heating in KSTAR H-mode plasmas
}

\author{
Joohwan Hong ${ }^{1,2}$, S. S. Henderson ${ }^{3}$, Kimin Kim ${ }^{1,2}$, C. R. Seon ${ }^{4}$, Inwoo Song ${ }^{1,2}$, H. Y. Lee ${ }^{1,2}$, \\ Juhyeok Jang ${ }^{1,2}$, Jae Sun Park ${ }^{1,2}$, S. G. Lee ${ }^{4}$, J. H. Lee ${ }^{4}$, Seung Hun Lee ${ }^{4}$, Suk-Ho Hong ${ }^{4}$, and \\ Wonho Choe ${ }^{1,2^{*}}$
}

${ }^{1}$ Department of Physics, Korea Advanced Institute of Science and Technology, 291 Daehak-ro, Yuseong-gu,
Daejeon 34141, Republic of Korea
${ }^{2}$ Impurity and Edge plasma Research Center, 291 Daehak-ro, Yuseong-gu, Daejeon 34141, Republic of Korea
${ }^{3}$ Department of Physics SUPA, University of Strathclyde, Glasgow G1 1XQ, UK
${ }^{4}$ National Fusion Research Institute, 169-148 Gwahak-ro, Yuseong-gu, Daejeon 34133, Republic of Korea

*Author to whom corresponding should be addressed; E-mail: wchoe@kaist.ac.kr

\begin{abstract}
Experiments with a small amount of Ar gas injection as a trace impurity were conducted in the Korea Superconducting Tokamak Advanced Research (KSTAR) H-mode plasma $\left(B_{T}=2.8 \mathrm{~T}, I_{P}=0.6 \mathrm{MA}\right.$, and $\left.P_{N B I}=4.0 \mathrm{MW}\right) .170 \mathrm{GHz}$ electron cyclotron resonance heating $(\mathrm{ECH})$ was focused along midplane with fixed major radial position of $R=1.66 \mathrm{~m}$ and scanned its power from 0 to $800 \mathrm{~kW}$. The emissivity of the $\operatorname{Ar}^{16+}(3.949 \AA)$ and $\operatorname{Ar}^{15+}$ (353.860 $\AA$ ) spectral lines were measured by the X-ray imaging crystal spectroscopy (XICS) and vacuum UV (VUV) spectrometer, respectively. The peak emissivity of $\mathrm{Ar}^{15+}$ was reduced by ECH, an effect largest with $800 \mathrm{~kW}$ compared to $600 \mathrm{~kW}$ of ECH. The $\mathrm{Ar}^{16+}$ emission increased with higher heating power. The ADAS-SANCO impurity transport code was used to evaluate the Ar transport coefficients. It was found that the inward convective velocity found in the plasma core without ECH was decreased with the $\mathrm{ECH}$, while diffusion remained approximately constant resulting in a less-peaked Ar density profile. Theoretical results from the NEO code suggest that neoclassical transport is not responsible for the change in transport, while the microstability analysis using GKW suggests a dominant ITG mode during both ECH and non-ECH plasmas.
\end{abstract}




\section{Introduction}

Impurities can have a detrimental effect on routine tokamak operation due to a number of reasons including core fuel dilution and radiation cooling. However, well-controlled injections of gaseous impurities can enhance the radiation near the divertor and therefore reduce the heat flux striking the plasma facing components. Controlling and understanding where both the intrinsic impurities, such as tungsten, and the injected impurities radiate their power and accumulate is therefore an important and critical issue for ITER and future fusion reactors. Previous experiments using auxiliary heating such as ECH or ICRH have observed reductions in both the concentration and accumulation of impurities in the plasma core [1-11]. In particular, our previous KSTAR experiments in L-mode plasmas demonstrated reversal of Ar impurity pinch direction by ECH [11]. More recent studies have made progress in the understanding of core impurity transport by including the centrifugal forces exerted on the heavy impurity ions [12,13], and also by laying emphasis on MHD behaviour [14,15]. However, there is still no clear explanation on the mechanisms involved in the changing transport induced by auxiliary heating, particularly the operational condition required to induce an outward convective velocity and thus hollow impurity profiles in the core.

Effects of ECH on impurity transport are investigated in this paper by analyzing trace Ar gas injection experiments whilst scanning the ECH power in KSTAR H-mode plasmas. Ar transport coefficients have been determined by SANCO [16] impurity transport code with measured $\mathrm{Ar}$ emission by spectroscopic diagnostics. Analysis results using neoclassical calculation by NEO [17,18] and gyrokinetic simulation by GKW [19] codes are presented to provide theoretical understanding of the role of ECH on the transport and core accumulation of Ar impurities in the H-mode plasma.

The paper is organized as follows. In section 2, the experimental condition and Ar emission measurements are described. Section 3 presents the analysis of Ar transport coefficients by SANCO and results from section 3 are compared with neoclassical and turbulence impurity transport calculations in section 4 . Finally, a summary is followed in section 5 .

\section{Experiment description and measurement results}

Experiments in KSTAR H-mode plasmas with 0.6 MA of plasma current, 2.8 T of central toroidal magnetic field and $4 \mathrm{MW}$ of NBI power were performed to investigate the effect of ECH on the Ar impurity transport. Two plasmas with an ECH power of 600 and $800 \mathrm{~kW}$ (Shot \#10649 and Shot \#10640, respectively) are compared to a reference plasma without ECH (Shot \#10647). For both ECH plasmas, the heating is focused on $\mathrm{Z}=0$ (mid-plane) and $\mathrm{R}=1.68 \mathrm{~m}$ (which corresponds to $r / a=0.3$ ) with $170 \mathrm{GHz} \mathrm{ECH}$. The exact heating position and the shape of plasma are shown in figure 1. Figure 2 depicts the temporal behaviour of the following plasma parameters for the non-ECH and $800 \mathrm{~kW}$ ECH cases: the plasma current, the line-integrated electron density, the core channel of the electron cyclotron emission diagnostics for the electron temperature, and the $\mathrm{Ar}^{15+}$ line-integrated emission. For each discharge, a trace amount of Ar gas was injected at $4.5 \mathrm{~s}$ during the plasma current flat-top phase by a piezo electric valve gas injector installed at the mid-plane. The duration of the gas puff was about $30 \mathrm{~ms}$, corresponding to injecting approximately $10^{18} \mathrm{Ar}$ atoms estimated by a calibrated gas flow meter. This trace amount of Ar impurity gas perturbs the plasma parameters, particularly electron density and temperature, by less than $10 \%$, but is enough to significantly perturb the intrinsic Ar line emission to separately determine the diffusion and convection transport coefficients [20]. ECH is applied at $0.5 \mathrm{~s}$ before the Ar injection allowing sufficient time to establish a steady electron temperature profile which is a condition for analysis of impurity particle transport. The time evolution of electron temperature in the core shows that there is no sawtooth behavior in every discharge. 


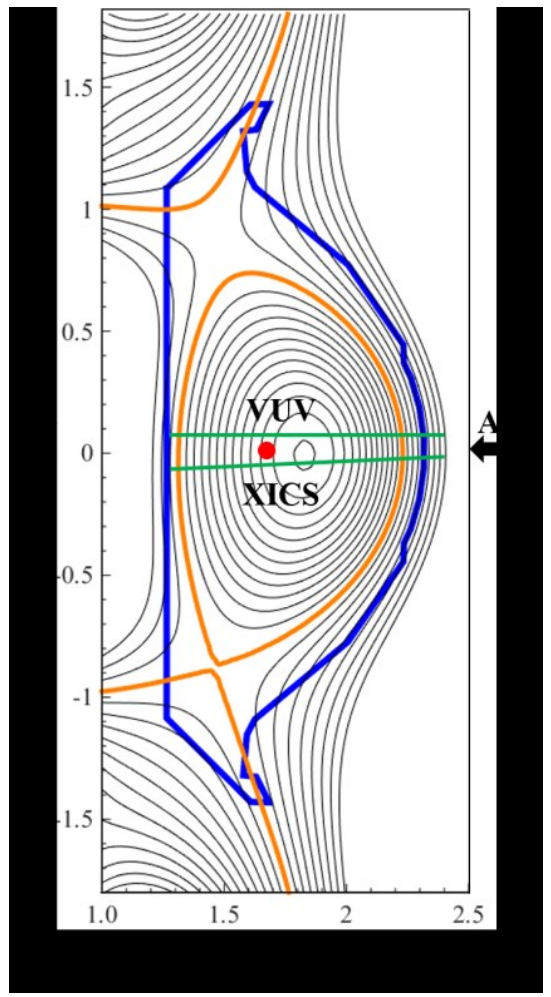

Figure 1. The plasma shape and line of sights of VUV and XICS spectrometers. The red dot is the heating position of ECH. The Ar gas was injected by a piezo value from the mid-plane.

(a)

(b)
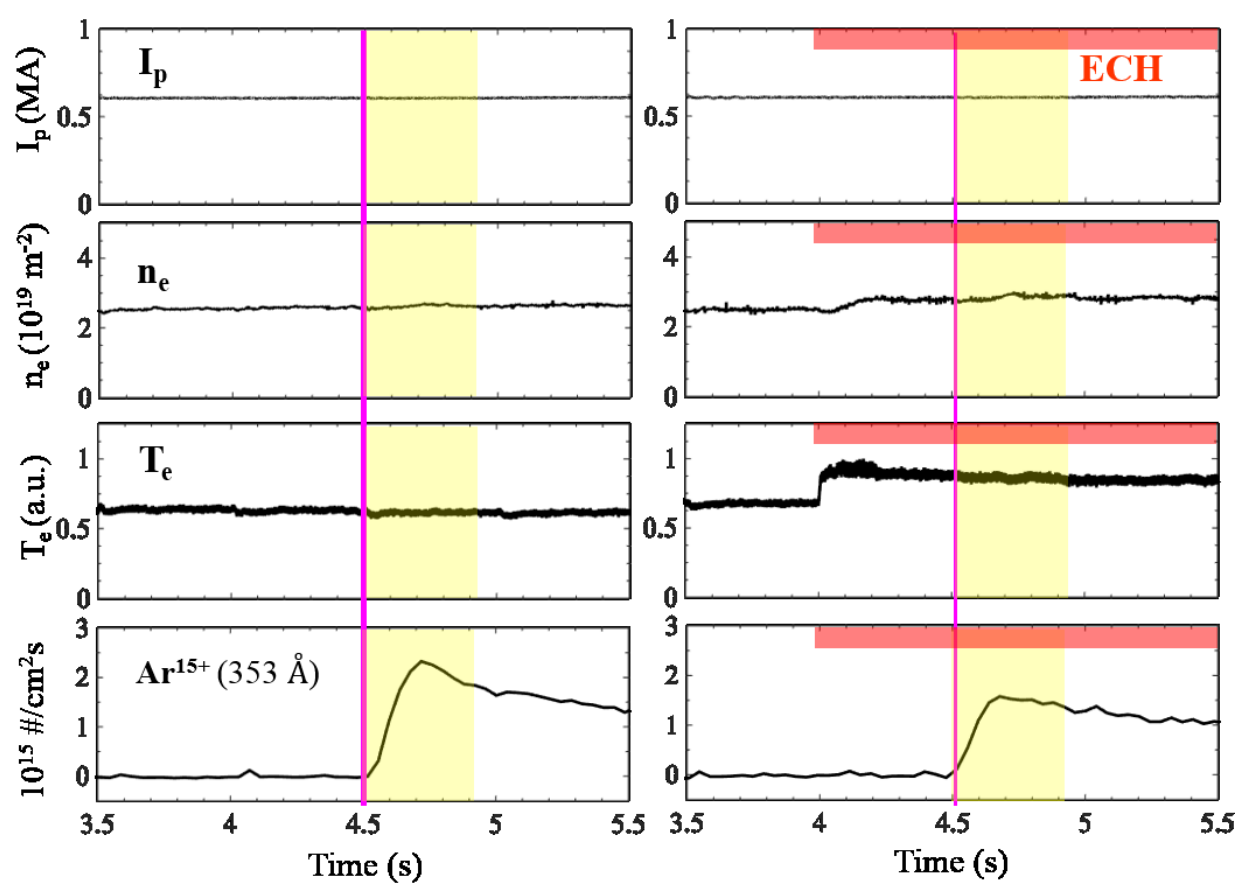

Figure 2. The time traces of plasma parameters for (a) non-ECH and (b) $800 \mathrm{~kW} E C H$ : plasma current $I_{P}$, line-integrated electron density $n_{e}$, electron temperature $T_{e}$, and $\mathrm{Ar}^{15+}$ emission from the VUV spectrometer. The red and yellow shaded regions represent the time-window of ECH and SANCO analysis, respectively. The vertical pink line is the Ar injection time (4.5 s). 
The radial profiles of electron temperature, electron density and ion temperature for each discharge are presented in figure 3. Electron temperature and density profiles were obtained from the Thomson scattering diagnostics and ion temperature profiles from charge exchange spectroscopy (CES). All profiles are averaged within the time-window of interest corresponding to the yellow shaded region in figure 2. At $5.0 \mathrm{~s}$, the NBI is off for $10 \mathrm{~ms}$ and therefore measurements from CES are not possible. Edge localized modes (ELMs) are always present, however they have a negligible effect on the time-averaged plasma profiles and the core Ar transport.

(a)

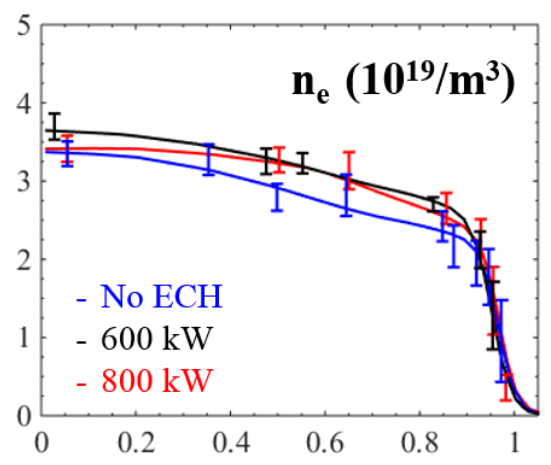

(b)

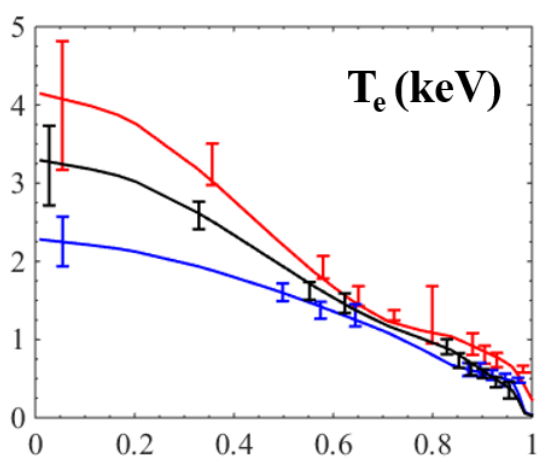

(c)

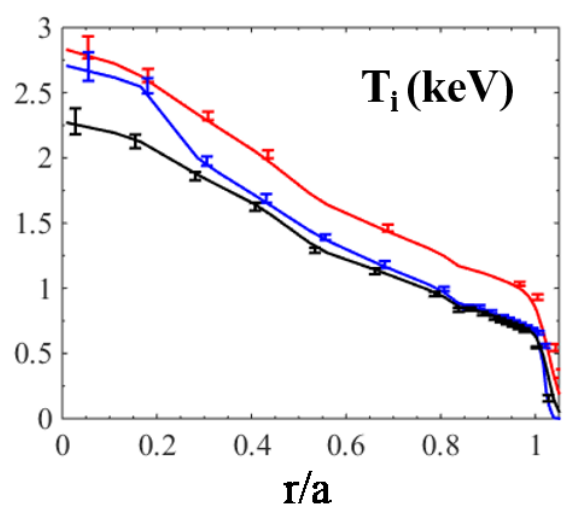

Figure 3. The radial profiles of (a) electron density, (b) electron temperature, and (c) ion temperature for non-ECH (blue), $600 \mathrm{~kW}$ ECH (black), and $800 \mathrm{~kW} \mathrm{ECH} \mathrm{(red).}$

Line emissions from the injected Ar ions have been measured using a vacuum ultra violet (VUV) spectrometer (130-600 ̊) [21] and an X-ray imaging crystal spectrometer (XICS) (3.93-4.00 ̊) [22]. The $\operatorname{Ar}^{15+}\left(353.860 \AA, 1 \mathrm{~s}^{2} 2 \mathrm{~s}^{2} \mathrm{~S}_{1 / 2}-1 \mathrm{~s}^{2} 2 \mathrm{p}^{2} \mathrm{P}_{3 / 2}\right)$ and $\mathrm{Ar}^{16+}\left(3.949 \AA \mathrm{A}^{16} 1 \mathrm{~s}^{2}{ }^{1} \mathrm{~S}_{0}-1 \mathrm{~s} 2 \mathrm{p}{ }^{1} \mathrm{P}_{1}\right)$ lines are used for the impurity transport analysis due to their strong intensity and quality of measurement. The time evolution of the two Ar line intensities are shown in figure 4. These measurements are integrated along the (green) sight lines shown in figure 1. 

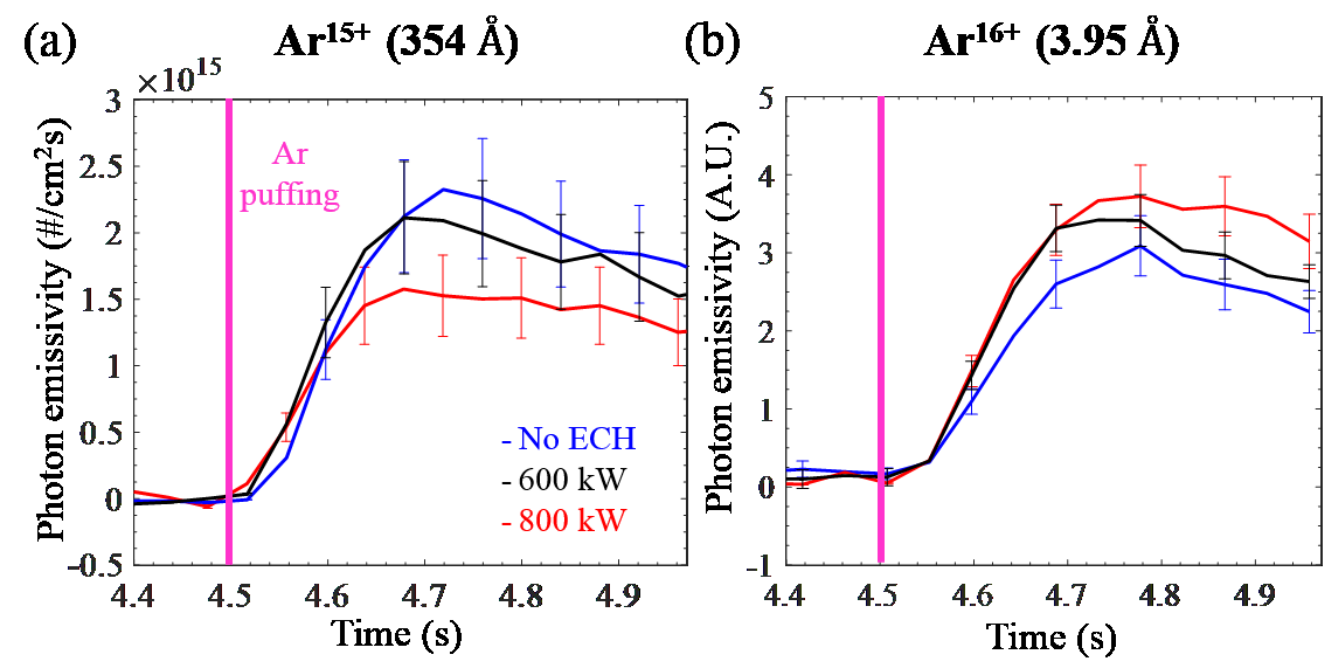

Figure 4. The time evolutions of (a) the $\operatorname{Ar}^{15+} 353 \AA$ and (b) the $\operatorname{Ar}^{16+} 3.95 \AA$ after Ar gas puffing at $4.5 \mathrm{~s}$

The measurements indicate that ECH reduces the peak emissivity of the $\mathrm{Ar}^{15+}$ emission and increases the $\mathrm{Ar}^{16+}$ emission. This trend was stronger with higher heating power. One should be cautious of immediately interpreting the changes in Ar line emission as a change in Ar transport because of the electron temperature and density dependence of the measured line emissivity which is calculated by:

$$
\epsilon_{z, j \rightarrow k}(t)=\int n_{e}(r, t) n_{z}(r, t) P E C_{z, j \rightarrow k}\left(T_{e}, n_{e}\right) d r
$$

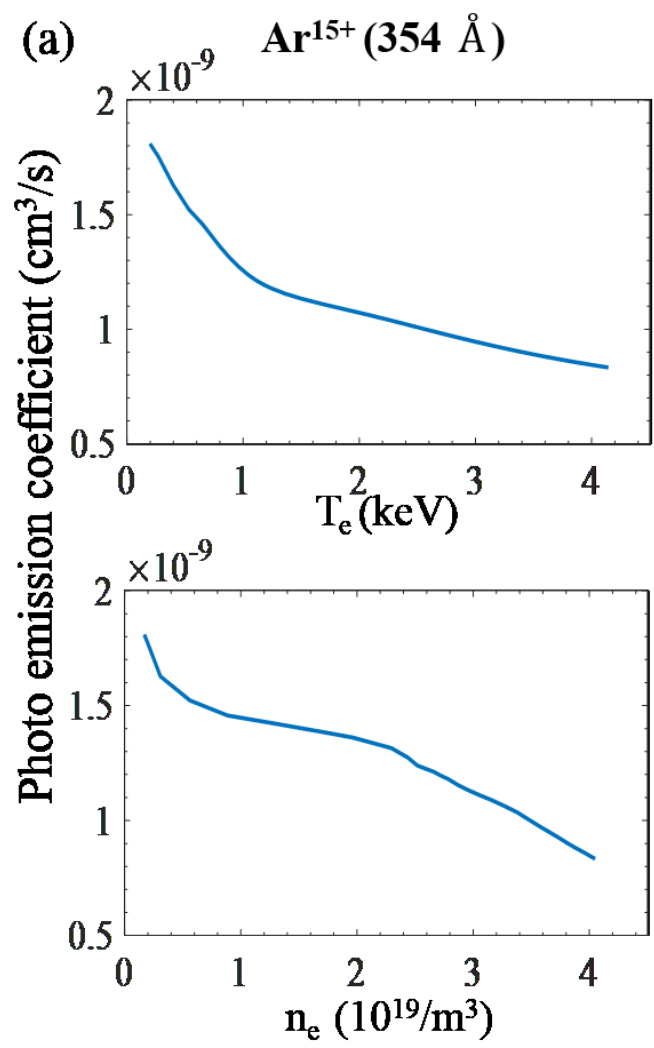

(b) $\times 10^{-11} \quad \operatorname{Ar}^{16+}(3.95 \AA)$
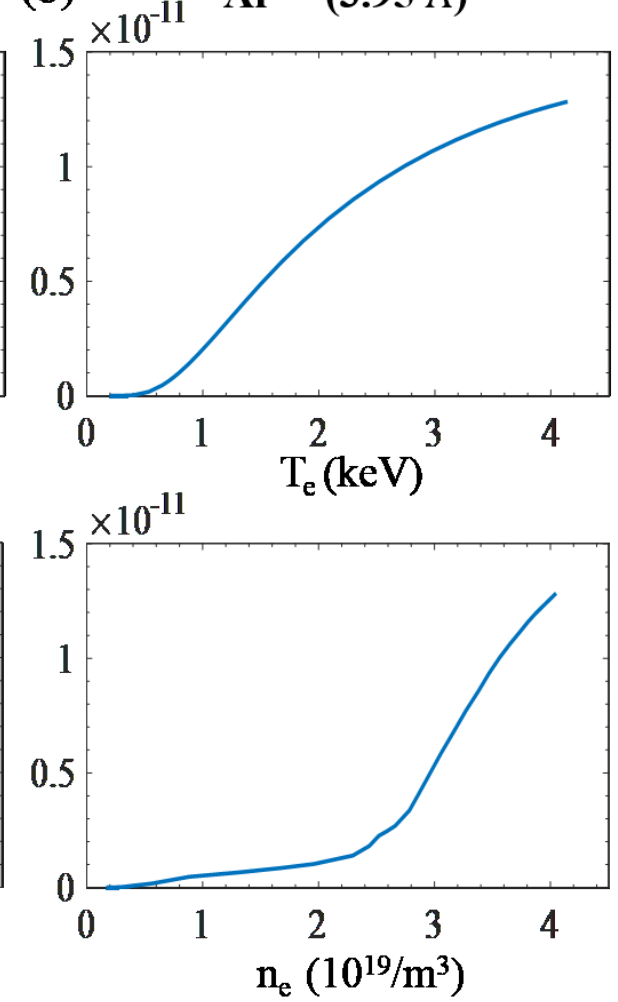
Figure 5. Photon emission coefficient of (a) the $\mathrm{Ar}^{15+} 353 \AA$ and (b) the $\operatorname{Ar}^{16+} 3.95 \AA$ versus electron temperature and density

where $n_{e}$ is the electron density, $n_{z}$ is the density of Ar with charge state $Z$, and the PEC is the photon emission coefficient from state $j$ to $k$ which is taken from the Atomic Data Analysis Structure (ADAS) [23]. The PEC of $353.860 \AA \mathrm{Ar}^{15+}$ and $3.949 \AA \mathrm{Ar}^{16+}$ are presented in figure 5. These results show that the $P E C$ of $\mathrm{Ar}^{15+}$ is inversely proportional to $T_{e}$ and $n_{e}$ while the $P E C$ of $\mathrm{Ar}^{16+}$ exhibits the opposite behaviour, which is also consistent with the measurement result shown in figure 4 . In reality, the difference in emission is a combination of both the change in impurity transport and the response of the emission to the change in the plasma equilibrium.

\section{Ar transport coefficients and radial distributions}

The 1.5 D radial impurity transport code SANCO, which is included in the fitting analysis code UTC [24], has been used to compare the measured evolution of the line-integrated Ar emission with a synthetic profile dependent on the radial Ar transport coefficients. SANCO solves the set of continuity equations for every charge state $Z$ of the impurity ions:

$$
\frac{\partial n_{Z}(r, t)}{\partial t}=-\nabla \cdot \Gamma_{Z}(r, t)+S_{Z}(r, t)
$$

where $n_{Z}$ and $\Gamma_{Z}$ are the density and the particle flux of the impurity ions, respectively. The $S_{Z}$ term includes the ionization and recombination coefficients from ADAS connecting neighboring charge states. The particle flux is usually expressed by the following ansatz consisting of two terms, a diffusion coefficient $D(r, t)$ and a convection velocity coefficient $V(r, t)$ :

$$
\Gamma_{Z}(r, t)=-D(r, t) \frac{\partial n_{Z}(r, t)}{\partial r}+V(r, t) n_{Z}(r, t)
$$

Transport coefficients $D(r, t)$ and $V(r, t)$ are assumed to be time independent since the main parameters of the plasma were approximately stationary during the analysis time window shown in figure 2. In addition, SANCO has a simplified edge model [25] containing a recycling coefficient and a parallel loss term. However, the edge model was turned off for this analysis because a peripheral Ar line measured by the visible spectrometer system was used to characterize the neutral influx.

The aforementioned two Ar lines were used for determining the transport coefficients. First, SANCO calculates radial density profiles of each ionization stage with the initial assumption of $D$ and $V$. The time evolution of Ar line emission can be modeled by equation (1) with time averaged $T_{e}$ and $n_{e}$ from figure 3. EFIT magnetic equilibrium profiles were used for the line integration process of local Ar emissions along the line-of-sight. The values of $D(r)$ and $V(r)$ were then adjusted iteratively by the least squares fitting code UTC until the synthetic emission reproduced the measured spectroscopic data with the minimum values of reduced $\chi^{2}$ close to unity in accordance with the experimental error bars. The absolutely calibrated measurements from the VUV spectrometer system on KSTAR provided a constraint on the prescribed Ar particle influx inside the last closed flux surface. On the other hand, the XICS system which measures $\mathrm{Ar}^{16+}$ line has not been calibrated absolutely, so it provides only relative intensities. Therefore, a normalization process has been conducted to derive the transport coefficient from XICS data.

The best fit results of the three discharges are presented in figure 6. Each column shows non$\mathrm{ECH}, 600 \mathrm{~kW} \mathrm{ECH}$ and $800 \mathrm{~kW} \mathrm{ECH}$ cases from top to bottom. Red lines are SANCO calculation result, and black bars indicate corresponding spectroscopic data with measurement errors. The 
determined $D(r), V(r)$ and peaking factor $V(r) / D(r)$ are plotted in figure 7. The main effects of $\mathrm{ECH}$ on transport
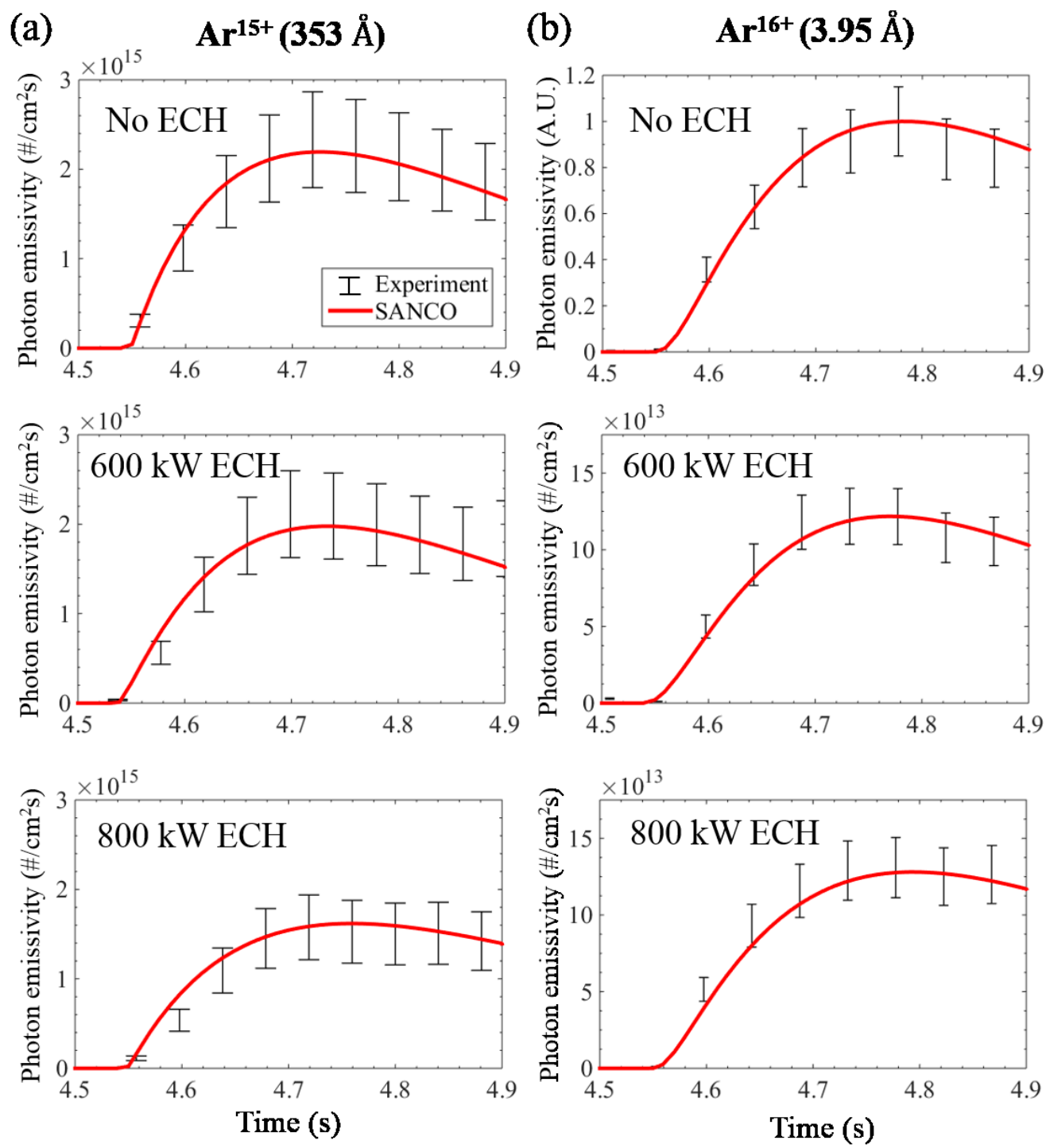

Figure 6. The SANCO analysis results for non-ECH, $600 \mathrm{~kW} \mathrm{ECH}$, and $800 \mathrm{~kW} \mathrm{ECH}$. Red curves represent the SANCO simulation result, and black dots with error bars show Ar emissivities of (a) the $\mathrm{Ar}^{15+}$ and (b) the $\mathrm{Ar}^{16+}$ lines

coefficients can be seen in $V(r)$ within $r / a<0.4$, where $a$ is the minor radius of the plasma. When applying ECH power, the magnitude of the inward convective velocity $(V<0)$ was decreased in the region of $r / a<0.4$. This trend was enhanced with higher ECH power. It is also noted that the direction of convection remained inward with $\mathrm{ECH}$, which is significant since $\mathrm{ECH}$ is often reported as a means for reversing the pinch direction from inward to outward in many tokamaks $[3,4,7,9,11]$. On the other hand, ECH caused a small increase in $D(r)$ across the plasma radius; however this was within the error bar. This weak modification of $D(r)$ by auxiliary heating has been observed as well in other impurity transport experiments $[10,26]$. The peaking factor $V(r) / D(r)$ follows the same trend as $V(r)$ in the region $r / a<0.4$ close to the heating position.

Using the transport coefficients detailed above, the time evolution of the total Ar density profile integrated over the plasma volume was calculated by SANCO for each discharge as shown in figure 8 . Every discharge shows that injected Ar particles were peaked in the core around $0.3 \mathrm{~s}$ after gas 
injection at $4.5 \mathrm{~s}$, regardless of applying ECH. At the time of peak accumulation, shown by the white dotted line in figure 8(a), the total and charge state Ar radial density profiles are presented in figure 8(b). The dominant charge state of Ar was He-like $\mathrm{Ar}^{16+}$ (magenta curve) and was present across the plasma radius, while $\mathrm{H}$-like $\mathrm{Ar}^{17+}$ ions (blue curve) existed mainly in the core and $\mathrm{Li}$-like $\mathrm{Ar}^{15+}$ ions (green curve) were distributed in the edge. Although a hollow Ar density profile was not achieved similar to the previous L-mode experiments in KSTAR [11], the Ar density and its gradient in the core was reduced with increasing power of $\mathrm{ECH}$.
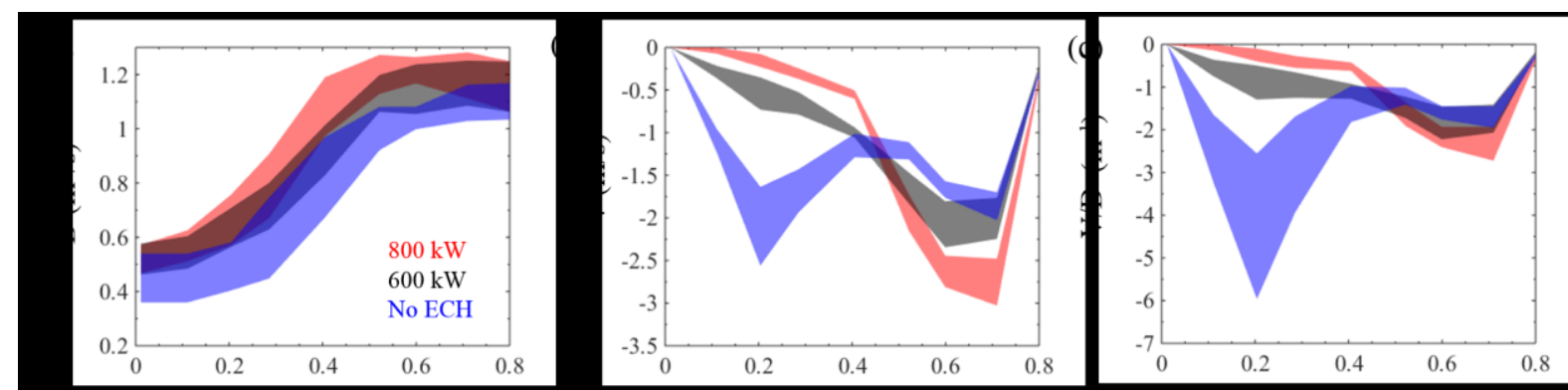

Figure 7. Radial profiles of transport coefficients for the non-ECH (blue), $600 \mathrm{~kW} \mathrm{ECH} \mathrm{(black)} \mathrm{and}$ $800 \mathrm{~kW}$ ECH (red): (a) diffusion $D$, (b) convection $V$, and peaking factor $V / D$
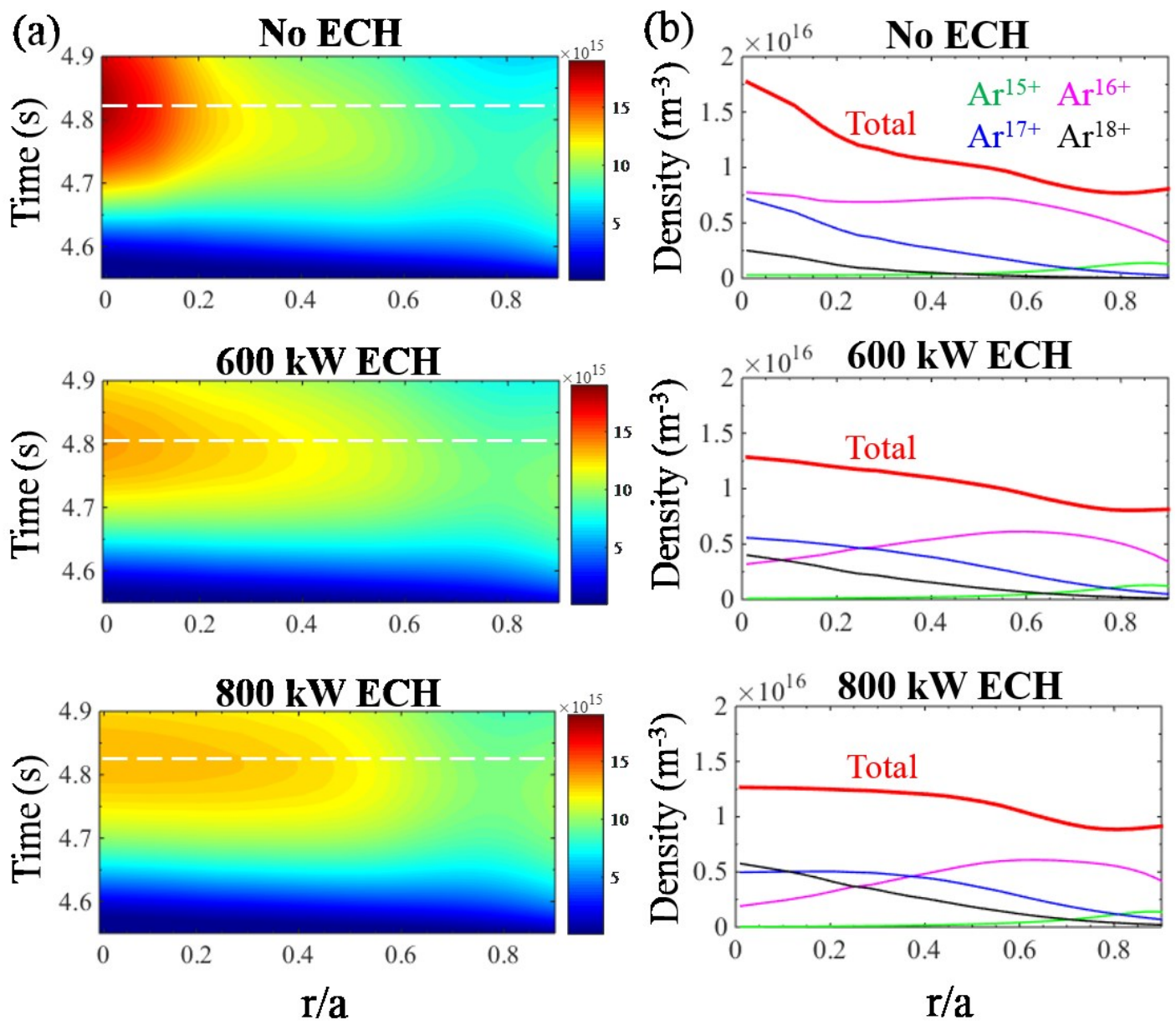

Figure 8. The SANCO simulation results of (a) the time evolution of radial distribution of total Ar density, and (b) radial Ar density profile of each charge state at peak time (white dotted line) 


\section{Theoretical impurity transport analysis}

The SANCO Ar impurity transport coefficients derived in the previous section are compared with neoclassical and turbulent predictions calculated using the local drift kinetic code NEO and the local flux-tube gyrokinetic code GKW. Plasma rotation obtained by CES measurements was also used in the calculation. The particle flux in both codes is calculated for a trace impurity $\mathrm{Ar}^{16+}$ because it is most abundant across the plasma radius as shown in figure 8(b). Figure 9 shows the neoclassical transport coefficients for the non-ECH and ECH cases. For every case, the neoclassical diffusion is 310 times smaller in magnitude compared to the SANCO diffusivity. Unlike the experiment, there is also little change in the neoclassical convective velocity within the plasma core. In conclusion, neoclassical transport cannot explain the experimental observation, and therefore turbulence analysis was performed to interpret the SANCO results.

(a) $\quad$ D $\left(\mathbf{m}^{2} / \mathbf{s}\right)$
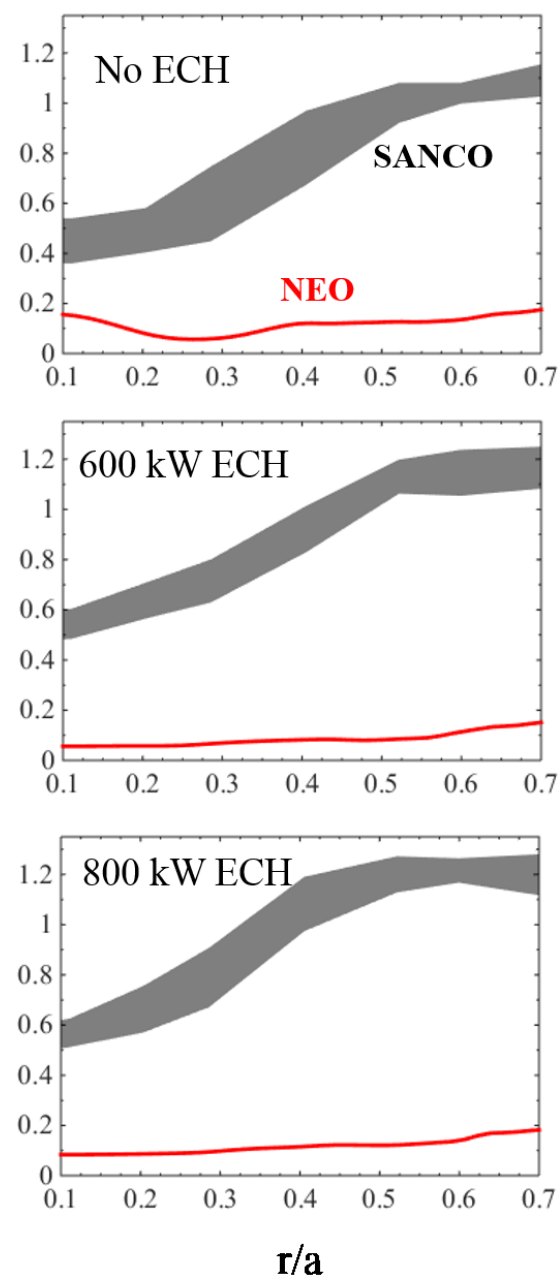

(b)
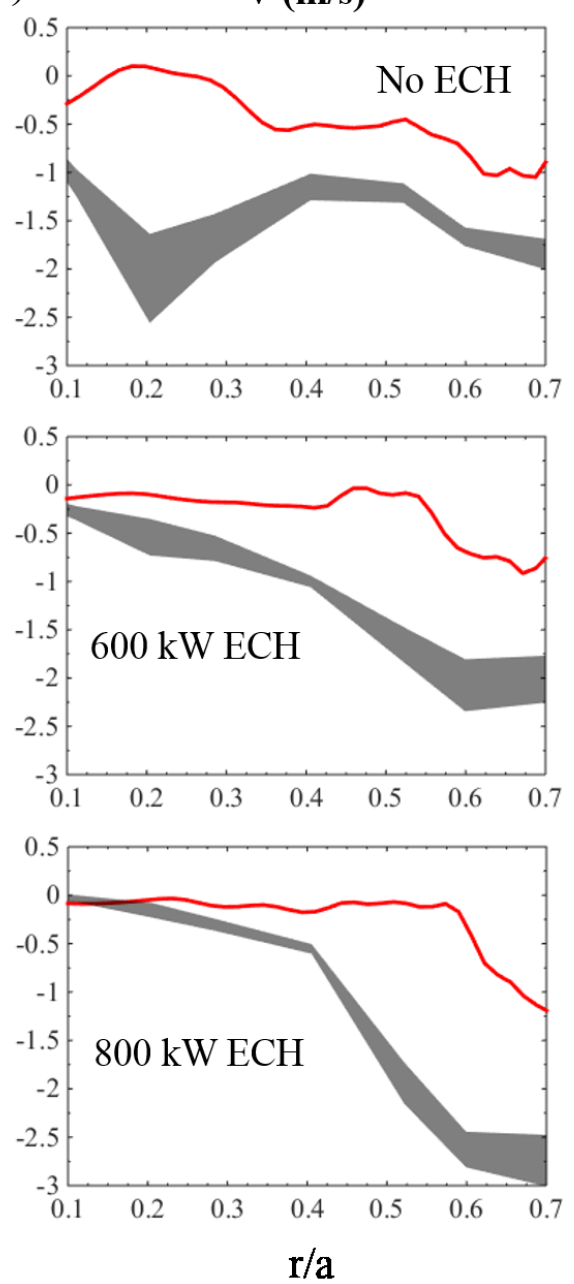

Figure 9. NEO calculations (red) of (a) $D$ and (b) $V$ profiles compared with the SANCO calculation (black) from figure 7

Local flux-tube electrostatic calculations at $r / a<0.3$ (where $r / a=0.3$ is the ECH target location) have been conducted using the input parameters listed in table 1: the logarithmic temperature and density gradients $R / L_{T_{e}}, R / L_{T_{i}}$, and $R / L_{n_{e}}$ (where $R$ is the major radius), the toroidal 
rotation gradient $u^{\prime}$, the safety factor $q$, and the magnetic shear $s$. The most significant change in the plasma equilibrium caused by $800 \mathrm{~kW} \mathrm{ECH}$ is an increase in $R / L_{T_{e}}$ and a decrease in $R / L_{T_{i}}$ while $R / L_{n_{e}}$ and $u^{\prime}$ slightly decrease, as shown in figure 3. To simplify the analysis, the ECH cases are divided into two cases: Case I taking only temperature changes into account, and Case II considering all changes listed above. In the GKW simulations, the electron temperature gradients obtained by electron cyclotron emission (ECE) diagnostics were used instead of the measurements from the Thomson scattering system because of their higher spatial resolution. The $q$ and $s$ factors were measured by the motional Stark effect (MSE) diagnostic system.

Table 1. Input parameters for GKW calculation for each case

\begin{tabular}{ccccccc}
\hline Case & $\boldsymbol{R} / \boldsymbol{L}_{\boldsymbol{T}_{\boldsymbol{e}}}$ & $\boldsymbol{R} / \boldsymbol{L}_{\boldsymbol{T}_{\boldsymbol{i}}}$ & $\boldsymbol{R} / \boldsymbol{L}_{\boldsymbol{n}_{\boldsymbol{e}}}$ & $\boldsymbol{u}^{\prime}$ & $\boldsymbol{q}$ & $\boldsymbol{s}$ \\
\hline Non-ECH & 1.87 & 5.5 & 1.5 & 1.45 & 1.85 & 0.63 \\
ECH plasma: Case I & 6.0 & 3.0 & 1.5 & 1.45 & 1.85 & 0.63 \\
ECH plasma: Case II & 6.0 & 3.0 & 1.0 & 1.0 & 1.85 & 0.63 \\
\hline
\end{tabular}

The results of the GKW analysis for the non-ECH and ECH Case I and II are illustrated in figure 10. Figures 10 (a)-(c) show the linear growth rate $\gamma$, the mixing length $\gamma / k_{y}^{2}$ (which is used to estimate the value $k_{y} \rho_{i}$ where the transport is maximum in the nonlinear saturated state [7]) and the mode frequency $\omega$ spectrum, respectively. Applying ECH causes a decay in the growth rate over the wavenumber range of $k_{y} \rho_{i}=0.1-1.0$ and a change in the peak position of $\gamma / k_{y}^{2}$. In all three cases, $\omega>0$ and the unstable modes are therefore rotating in the ion diamagnetic direction. Therefore, ion temperature gradient (ITG) modes were most dominant during the experiments, regardless of ECH. This result is also consistent with other experiments which reported negligible change in the impurity diffusivity when ITG remained the dominant mode after applying ECH [26] and ICRH [10]. It is also noted that the experiments that observed a significant modification of diffusion with additional heating also reported a dominant mode transition from ITG to trapped electron mode (TEM) [6] or a reversal of the propagation direction of the most unstable mode [7].The turbulent $\mathrm{Ar}^{16+}$ peaking factor is evaluated with GKW using the quasi-linear approach described previously [27, 28] which showed good agreement with non-linear gyrokinetic simulations [29]. However, in these previous cases, the analysis focuses on estimating the low field side peaking factor to correspond to their experimental (charge exchange) measurements. In this analysis, the transport coefficients were deduced from (midplane) line-integrated measurements and therefore represent flux-surface averaged quantities. Due to the heavy mass of Ar, there is likely a significant poloidal variation in the Ar density distribution [28], meaning that the flux-surface averaged and low field side gradients are not equal.

To calculate the flux-surface averaged peaking, firstly two trace impurity species, each with different (arbitrary) radial gradients, are included in the gyrokinetic simulations. It is important to provide these two trace impurities with accurate temperature and toroidal rotation gradients to correctly account for changes in the associated particle flux caused by centrifugal effects [30]. The diffusion and convective velocity coefficients are then respectively calculated as the gradient and yintercept of the line

$$
\frac{R\left\langle\Gamma_{Z}\right\rangle}{\left\langle n_{Z}\right\rangle}=\left\langle D_{Z}\right\rangle\left\langle\frac{R}{L_{n}}\right\rangle+\left\langle V_{Z}\right\rangle
$$

where $\left\langle R / L_{n}\right\rangle$ is calculated internally by GKW for both trace impurity species and provided as an output. The individual contributions to the peaking factor $V / D$ from thermo-diffusion $C_{T}$ [31], rotodiffusion $C_{u}$ [32], and pure convection $C_{p}$ [33] can be determined by including two additional trace 
impurity species, one with $u^{\prime}=0$ and the other with $R / L_{T_{e}}=0$.

The peaking factors and individual contributions are shown for the three cases listed above in figures 10 (d)-(f). The shaded regions indicate the value of $k_{y}$ associated with the dominant linear mode. In all three cases, the peaking factor is determined by the balance of the inward pure convective term and the outward roto-diffusive term, while thermo-diffusion has a negligible effect on the peaking factor. For the non-ECH case, the theoretical peaking factor is approximately $1.6 \mathrm{~m}^{-1}$, which is within the experimental prediction of $2.65 \pm 1.14 \mathrm{~m}^{-1}$. Although the theoretical peaking factor is found to decrease to a value of about $0.8 \mathrm{~m}^{-1}$ in Case I, where $R / L_{T_{e}}$ is increased and $R / L_{T_{i}}$ is decreased, it is not found to agree within the experimental value of $0.42 \pm 0.13 \mathrm{~m}^{-1}$. The lack of agreement in magnitude is exacerbated in Case II (lower $R / L_{n_{e}}$ and $u^{\prime}$ ) where a peaking factor of about $1.9 \mathrm{~m}^{-1}$ is found. It is also noted that the values of $C_{u}$ and $C_{p}$ change significantly in all three cases, however the ratio of the two terms remains approximately constant.

(a) Growth rate
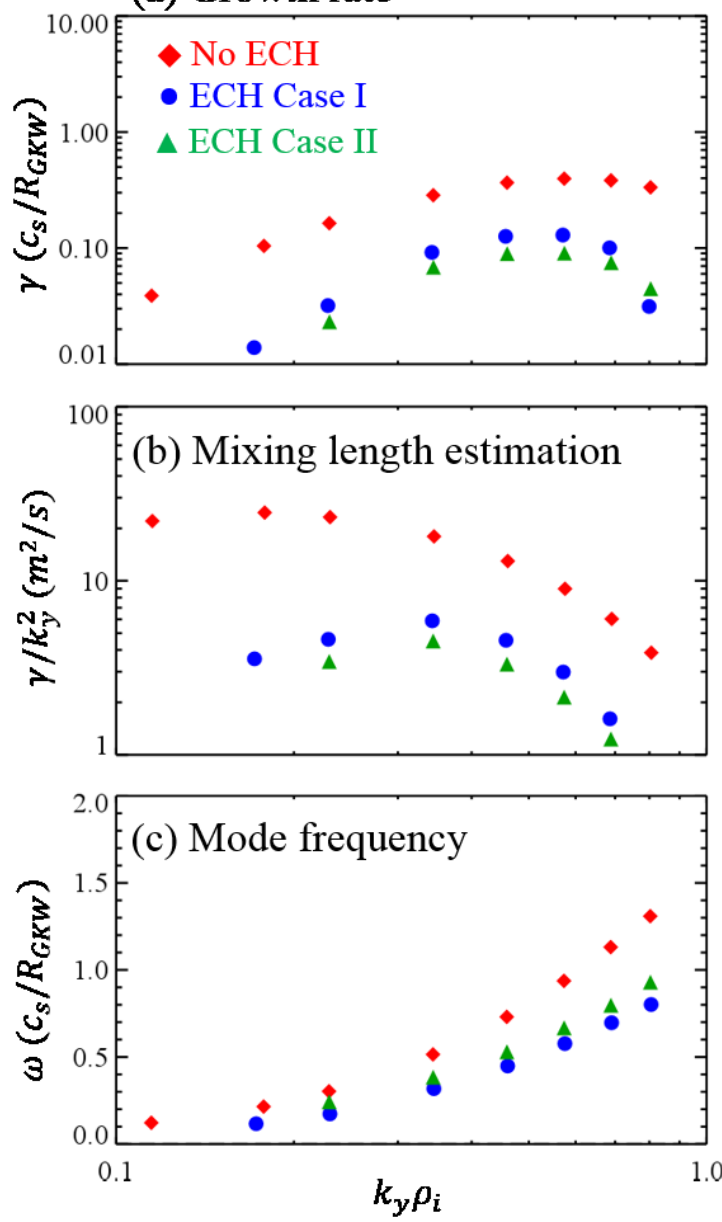

(d) No ECH
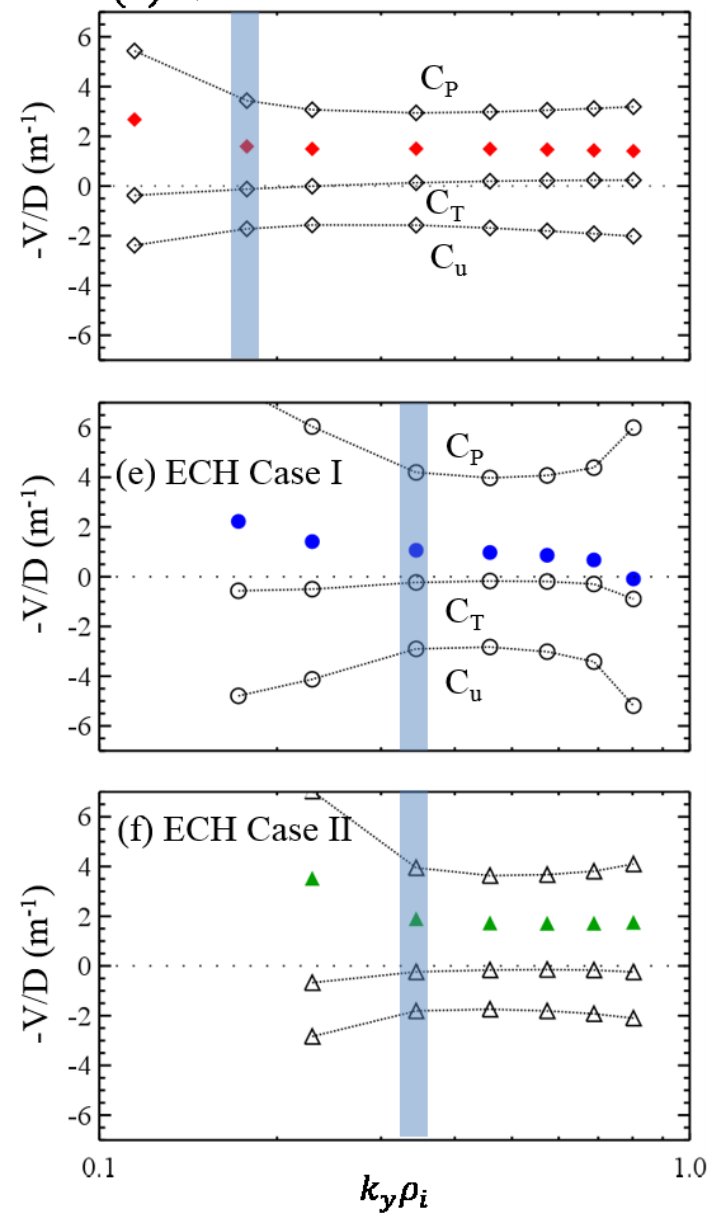

Figure 10. GKW simulation results for the no ECH (red), ECH Case I (blue) and II (green). (a) Linear growth rates, (b) mixing length estimation, and (c) mode frequency. (d)-(f) show that flux surface averaged peaking factors and individual contributions from thermo-diffusion $C_{T}$, rotodiffusion $C_{u}$, and pure convection $C_{p}$. The blue shaded regions represent the value of $k_{y}$ associated with the dominant linear mode.

In summary, the modification of the diffusivity by additional heating is thought to be negligible when the dominant turbulent mode propagates in the ion diamagnetic direction. Conversely, it appears 
that there is no clear relation between the direction of the convective velocity and the propagation direction of the most unstable turbulent mode. A reversal of pinch direction by additional heating which provides a hollow profile of impurities has been observed when dominant mode was TEM with ECH [11] or ICRH [7]. However, an exceptional observation, the pinch reversal by ICRH under ITG environment case [10], has also been reported. Further studies on a condition for pinch direction reversal need to be conducted, since an outward convection of impurities is the key to suppress and control of the core accumulation of impurity. For quasi-linear peaking factor calculation results, although a good agreement between the quasi-linear and SANCO peaking factor is found in the discharge without $\mathrm{ECH}$, the lack of change in the quasi-linear peaking factor when the temperature, density and toroidal rotation gradients are altered to match the plasma response to ECH in H-mode suggests that further investigation into the gyrokinetic particle flux is also required. In particular, when the turbulent mode remains dominantly ITG during additional heating, a discrepancy between gyrokinetic calculation and experiments has been reported [10] like our study.

\section{Summary}

Experiments with trace injections of Ar gas have been conducted in KSTAR H-mode discharges with and without ECH to investigate effects of additional heating on Ar impurity transport and its accumulation. The experiments are implemented by changing the heating power of ECH, while measuring the temporal Ar line intensities from the VUV and XICS signals. ECH reduced the $\mathrm{Ar}^{15+}$ emission and increased the $\mathrm{Ar}^{16+}$ emission. Both of these trends were amplified with increasing heating power.

These observations were interpreted in terms of the Ar particle diffusivity and convective velocity coefficients using the SANCO impurity transport code. Applying ECH did not significantly change the Ar diffusivity outside the fitting error bars, but did cause a reduction in the inward convective velocity within the plasma core. This change in transport therefore lowered the peaking factor $V / D$ and reduced the amount of Ar within the heating position $(r / a=0.3)$. It is noted that the direction of the convective velocity around the heating position was inward in both ECH and non-ECH plasmas; it did not reverse with ECH as shown previously in KSTAR L-mode plasmas [11].

The NEO and GKW codes were used to calculate the flux surface averaged neoclassical particle flux and quasi-linear Ar peaking factor, respectively. From this it was shown that the neoclassical transport coefficients were 3 to 10 times smaller than the SANCO results regardless of ECH power. Local flux-tube electrostatic calculations by GKW firstly showed that ITG was the dominant turbulent mode at $r / a=0.3$ for every case. This result is consistent with previous experiments [26] that found negligible changes in diffusivity when the turbulent modes propagate in the ion diamagnetic direction. This also suggests that TEM turbulence is more effective at enhancing the impurity diffusivity and hence reducing the core accumulation. The flux surface averaged Ar peaking factor was calculated by adopting a quasi-linear approach and was found to agree within the experimental error for the non$\mathrm{ECH}$ case, but overestimated the peaking factor for the ECH case.

In conclusion, this study has demonstrated that off-axis ECH reduces the core Ar accumulation in KSTAR H-mode plasmas by reducing the core inward convection. Further experiments scanning the heating position of ECH and injecting different impurities, such as $\mathrm{Kr}$ and $\mathrm{W}$, will be conducted in KSTAR to clarify the experimental conditions required to minimize the core impurity accumulation using $\mathrm{ECH}$, with particular focus on the direction of the convective velocity.

\section{Acknowledgements}


The authors are grateful to Drs. S.A. Sabbagh and Young-Seok Park of Columbia University for providing EFIT magnetic reconstruction data and to KSTAR team, especially Drs. H. H. Lee and W. Ko for CES analysis, J. W. Yoo for XICS data, S. H. Son for visible spectroscopy data and Dr. Jinseok Ko for MSE data. The authors would also like to thank Drs. Jeff Candy and Emily Belli for providing NEO code and Dr. Francis Casson for his help using the GKW code. This work was supported by the National Research Foundation of Korea (NRF), funded by the Ministry of Science, ICT and Future Planning (NRF-2014M1A7A1A03045092).

\section{References}

[1] Neu R., Dux R., Geier A. et al 2002 Plasma Phys. Control. Fusion 44811

[2] Puiatti M. E., Mattioli M., Telesca G. et al 2002 Plasma Phys. Control. Fusion 441863

[3] Takenaga H, Higashijima S., Oyama N. et al 2003 Nucl. Fusion 431235

[4] Dux R, Neu R., Peeters A. G. et al 2003 Plasma Phys. Control. Fusion 451815

[5] Puiatti M. E., Valisa M., Mattioli M. et al 2003 Plasma Phys. Control. Fusion 452011

[6] Puiatti M. E., Valisa M., Angioni C. et al 2006 Phys. Plasmas 13042501

[7] Angioni C., Dux R., Fable E. et al 2007 Plasma Phys. Control. Fusion 492027

[8] Leigheb M., Romanelli M., Gabellieri L. et al 2007 Plasma Phys. Control. Fusion 491897

[9] Sertoli M., Angioni C., Dux R. et al 2011 Plasma Phys. Control. Fusion 53035024

[10] Valisa M., Carraro L., Predebon I. et al 2011 Nucl. Fusion 51033002

[11] Hong J., Lee S. H., Kim J. et al 2015 Nucl. Fusion 55, 063016

[12] Angioni C., Mantica P., Pütterich T. et al 2014 Nucl. Fusion 54083028

[13] Casson F. J., Angioni C., Belli E. A. et al 2015 Plasma Phys. Control. Fusion 57014031

[14] Sertoli M., Dux R., Pütterich T. et al 2015 Plasma Phys. Control. Fusion 57075004

[15] Sertoli M., Odstrcil T., Angioni C. et al 2015 Nucl. Fusion 55113029

[16] Lauro-Taroni L. et al 1994 21st EPS Conf. on Controlled Fusion, Plasma Physics (Montpellier) p 102 I

[17] Belli E. A., Candy J. 2008 Plasma Phys. Control. Fusion 50095010

[18] Belli E. A., Candy J. 2012 Plasma Phys. Control. Fusion 54015015

[19] Peeters A. G., Camenena Y., Casson F. J. et al 2009 Comput. Phys. Commun. 1802650

[20] Marmar E. S., Cecchi J. L., Cohen S. A. 1975 Rev. Sci. Instrum. 461149

[21] Seon C. R., Hong J., Jang J. et al 2014 Rev. Sci. Instrum. $8511 \mathrm{E} 403$

[22] Lee S. G., Bak J. G., Nam U. W. et al 2007 Rev. Sci. Instrum. 78063504

[23] Summers H. P. 2004 The ADAS User Manual, version 2.6 http://www.adas.ac.uk

[24] Whiteford A. 2004 'On the spectral emission of impurity species for diagnostic application to magnetically confined fusion plasmas', Ph.D. Dissertation, University of Strathclyde, Glasgow, U.K.

[25] Giroud C., Barnsley R., Buratti P. et al 2007 Nucl. Fusion 47313

[26] Villegas D., Guirlet R., Bourdelle C. et al 2014 Nucl. Fusion 54073011

[27] Henderson S. S., Garzotti L., Casson F. J. et al 2014 Nucl. Fusion 54093013

[28] Henderson S. S., Garzotti L., Casson F. J. et al 2015 Plasma Phys. Control. Fusion 57095001

[29] Casson F. J., McDermott R.M., Angioni C. et al 2013 Nucl. Fusion 53063026

[30] Angioni C., Casson F. J., Veth C., Peeters A. G. 2012 Phys. Plasmas 19122311

[31] Coppi B., Spight C. 1978 Phys. Rev. Lett. 41551

[32] Camenen Y., Peeters A. G., Angioni C. et al 2009 Phys. Plasmas 16012503

[33] Angioni C., Peeters A. 2006 Phys. Rev. Lett. 96095003 
